\title{
Association of British Clinical Diabetologists (ABCD) position statement on the use of biosimilar insulin
}

\author{
VIJAY JAYAGOPAL, ${ }^{1}$ RUSSELL DRUMMOND, ${ }^{2}$ DINESH NAG| ${ }^{3}$ ON BEHALF OF THE ABCD
}

\begin{abstract}
Biosimilar insulins have the potential to offer the NHS a considerable cost saving. The acceptability and use of biosimilar insulin by clinicians and patients may, however, be limited by lack of experience, understanding and concerns about safety and tolerability. This article summarises information on the advantages and disadvantages of using biosimilar insulins and an overview of the published evidence in relation to efficacy, tolerability and safety of current and expected Biosimilar Insulins. The position of the ABCD on the use of Biosimilar Insulin is stated along with the key practical considerations for healthcare staff involved in insulin prescription and administration.

Br J Diabetes 2018;18:171-174
\end{abstract}

Key words: biosimilar insulin, safety, immunogenicity, switch

\section{Introduction}

Biosimilar insulins have the potential to offer the NHS a considerable cost saving and are therefore of significant interest to most area prescribing committees. Their acceptability and use by clinicians and patients may, however, be limited by lack of experience, understanding and concerns about safety and tolerability. The potential for cost saving is very real and important for the NHS, but must be balanced against the cautions and limitations of the use of biosimilar insulins. It is incumbent upon the healthcare teams supporting people with diabetes to understand fully these issues to ensure the correct choice is made for each individual patient. There is the additional - but less often acknowledged - complexity for healthcare staff of the proliferation in insulin products leading to confusion and medication errors. These are real risks that will need to be managed and mitigated whilst considering the adoption of new insulins or biosimilar products in terms of efficacy or cost advantages. This

York Teaching Hospitals NHS FT, UK

North Glasgow Diabetes and Endocrinology, Glasgow, UK

Edna Coates Diabetes and Endocrine Unit, Pinderfields Hospital, MYT, UK

Address for correspondence: Dr Vijay Jayagopal

Consultant Physician and Clinical Dean, Hull York Medical School, York Teaching Hospitals NHS FT, Wigginton Road, York YO31 8HE, UK

Tel: +44 (0)1904 721175

E-mail: Vijay.Jayagopal@York.NHS.UK

https://doi.org/10.15277/bjd.2018.190 position statement has been produced to try and help provide some clarity on the use, safety and current guidance on biosimilar insulins in the UK.

\section{What is a biosimilar?}

A biosimilar is a biological medicinal product that is developed to be highly similar to an existing biological medicine (also referred to as the reference medicine) in physiological and biological terms. Unlike most inorganic drugs that have simple chemical structures that allow for relatively simple replication as generic drugs or identical copies, biological drugs are complex molecules. The size and complexity of a biosimilar means an exact copy of the original cannot be guaranteed. ${ }^{1}$ Modified biopharmaceutical agents have been approved for clinical use since 1982 when the FDA sanctioned the introduction of recombinant human insulin. ${ }^{2,3}$ Biosimilar products have been developed and used in clinical practice for a number of clinical indications; examples are erythropoietin, growth hormone and monoclonal antibodies. To gain regulatory approval, the biosimilar insulin must show that any differences between it and the original insulin are not clinically meaningful in terms of quality, efficacy and safety. Detailed guidance on the approval process for biosimilar medicinal products by regulatory bodies has been produced. ${ }^{4}$ Once it is demonstrated to be comparable, the biosimilar is assumed to have the same benefits and risks as the original insulin in terms of quality, efficacy and safety. ${ }^{4,5}$

\section{Advantages and disadvantages of biosimilar insulin}

The main advantage of biosimilar insulin is the potential cost discount compared with the original insulin of about 15\%. While this is less of a discount than traditional generic drugs versus originator molecules, it does provide an opportunity for substantial cost reduction when translated across the NHS. A real problem in diabetes care provision is the lack of knowledge among healthcare professionals of the various insulins and the delivery devices linked to specific insulins. Biosimilar insulins add to this complexity. The increasing number and types of insulins, both original and biosimilar, will prove to be a major challenge in relation to avoiding both prescription and dispensing errors. Healthcare staff will need training and guidance on which insulins can be used interchangeably and where specific devices are needed.

Immunogenicity is another important safety concern as any biological agent can induce the formation of antibodies. This can manifest as serious allergic reactions, local injection site reactions or as effects on glucose lowering and required insulin dose. In 
the ELEMENT 1 and 2 studies ${ }^{6,7}$ the incidence of allergic reactions was similar in both treatment groups and the immunogenicity profiles were comparable. In ELEMENT 1, a total of $39.8 \%$ had detectable antibodies to insulin at 52 weeks and, in ELEMENT $2,13.2 \%$ had detectable antibodies to insulin at 24 weeks. There was no statistically significant difference seen between the two groups and there is no evidence that these antibodies had any impact on efficacy and safety outcomes.

In both ELEMENT 1 and ELEMENT 2 studies, the number of people with adverse events was similar in both groups, particularly in relation to hypoglycaemic episodes. 6,7 Immunogenicity data for other biosimilar insulins tested are also similarly reassuring. ${ }^{8-10}$

\section{Interchangeability and substitution}

Whether the original insulin and the biosimilar can be used interchangeably is an important consideration. This is because switching between the two may occur either by deliberate substitution (policy decision to switch) or accidental substitution at the time of dispensing or administration.

Currently, the only biosimilar insulin approved and available for clinical use is Abasaglar. The European Public Assessment Report on biosimilar insulin stated that, from the data provided from the studies on Abasaglar, where patients were switched from Lantus to Abasaglar at the same dose regimen, no difference in dose changes after titration to tighten blood glucose control was seen between the two treatment arms.

However, the following points are important to remember:

- The delivery device is different and the Abasaglar device is not the same as that of Lantus.

- Abasaglar cartridges cannot be used in the re-usable pen devices produced for Lantus.

- All biological medicines, including biosimilars, must be prescribed by brand name to prevent automatic substitution at the point of dispensing. ${ }^{5}$

- Abasaglar should only be initiated in patients new to insulin glargine or in those who require a review of their therapy due to poor control.

- Those who are stable on Lantus should not be switched to Abasaglar.

This guidance may well alter going forward once more clinical data are available, particularly from post-marketing and surveillance analysis.

\section{Review of data on biosimilar insulin}

\section{Insulin Glargine biosimilar Abasaglar}

The biosimilar development pathway for Abasaglar followed the prescribed comparability exercise to the original Lantus and the data submitted included initial phase I studies on healthy individuals as well as people with type 1 diabetes..$^{11,12}$ These demonstrated comparable bioavailability, pharmacokinetic (PK) and pharmacodynamic (PD) properties for Abasaglar and Lantus. The clinical comparability of Abasaglar to Lantus was based on phase III studies in people with type 1 diabetes (ELEMENT 1) ${ }^{6}$ and type 2 diabetes (ELEMENT
2). ${ }^{7}$ In both studies, once-daily Abasaglar was non-inferior to oncedaily Lantus (primary end point) and Lantus was non-inferior to Abasaglar (secondary end point), demonstrating equivalent efficacy of both medicines.

There are no published clinical studies comparing Abasaglar with Lantus in children and young adults. This is not a requirement, however, for the biosimilar approval process and the summary of product characteristics for Abasaglar includes reference to paediatric studies using Lantus where the efficacy and safety has already been established. The European Commission (EC) approved the marketing authorisation for Abasaglar on 9 September 2014 based on the submitted evidence.

\section{Other biosimilar insulins expected to be available in the near future}

The EC has approved marketing authorisation for two additional biosimilar insulins in 2017 and the European Medicines Agency (EMA) has published supporting European Public Assessment Reports which are briefly discussed here.

\section{Insulin Glargine Biosimilar Lusduna}

The marketing authorisation for insulin Lusduna was granted on 4 January 2017 for indications similar to that of basal insulin Lantus. The PK and PD were studied for biosimilar comparability to Lantus and adequately demonstrated bioequivalence for Lusduna versus Lantus with similar time action profiles and no unexpected or concerning toxicology results. ${ }^{8}$

The clinical similarity data are based on two phase III studies comparing Lusduna to Lantus. At 24 weeks there was no significant difference between Lusduna and Lantus in the reduction in $\mathrm{HbA}_{1 \mathrm{c}}$ seen in type 1 and in type 2 diabetes. In addition, similar changes from baseline were observed for people treated with Lusduna and Lantus in relation to other parameters measured such as insulin dose, mean 7-point self-monitoring of blood glucose and $\mathrm{HbA}_{1 \mathrm{c}}$ at goal of $<7.0 \%(53 \mathrm{mmol} / \mathrm{mol})$. The analysis of adverse events and severe adverse events, including specifically hypoglycaemia, found no meaningfully significant differences between the groups treated with Lusduna or Lantus.

\section{Sanofi Biosimilar Insulin Lispro}

The marketing authorisation for Biosimilar Insulin Lispro (BIL) was granted on 19 July 2017 for indications similar to those already established for Insulin Humalog. This is the first biosimilar prandial insulin to receive authorisation and maintained the regulatory approval pathway similar to other biosimilar insulins, but with studies designed specifically to assess data on postprandial control in addition to a reduction in $\mathrm{HbA}_{1 \mathrm{c}}$ and safety data.

The PK and PD analysis was supportive of BIL being of comparable clinical pharmacology to that of Humalog. ${ }^{9}$

Two primary clinical phase III regulatory studies were performed, one in people with type 1 diabetes (SORELLA 1) and the second in type 2 diabetes (SORELLA 2). After 26 weeks of treatment, the results indicated a similar change in $\mathrm{HbA}_{1 \mathrm{c}}$ between BIL and Humalog in both studies. Similarly, in both studies there were no significant differences noted in the secondary clinical outcomes 
studied (similarity in immunogenicity, relationship of anti-insulin antibodies and safety) between BIL and Humalog. Adverse events including hypoglycaemia were similar in those treated with BIL and Humalog.

There was a smaller additional study in people with type 1 diabetes using continuous subcutaneous insulin infusions (CSII) focusing on safety with the primary end point here being the incidence of set occlusions. No significant differences were noted between those using BIL and Humalog in the CSII study. Injection site reactions were rare and both groups had similar reported rates of hypersensitivity reactions. ${ }^{9}$

\section{Mylan Biosimilar Insulin Glargine (Semglee)}

The Committee for Medicinal Products for Human Use recommended approval of the use of Semglee on 25 January 2018. Details of the guidance will be made available in a European public assessment report after the EC formally grants marketing authorisation.

The mainstay of the application is data from the two INSTRIDE non-inferiority studies comparing MYL-1501D versus Lantus in people with type 1 and type 2 diabetes. In both studies the primary endpoint was change from baseline in $\mathrm{HbA}_{1 \mathrm{c}}$ after 24 weeks. Secondary endpoints included glycaemic endpoints such as change from baseline in fasting plasma glucose and insulin dose. ${ }^{10}$

\section{$A B C D$ position}

$A B C D$ acknowledges the benefit from the development of biosimilar insulins predicated upon potential cost savings to the NHS without compromising either efficacy or safety. Biosimilar insulins could be considered for all newly diagnosed patients with type 1 diabetes who have not been exposed to the reference drug and in patients who require a review of their therapy due to poor control. When patients are established on a current insulin regimen, those achieving their target $\mathrm{HbA}_{1 \mathrm{c}}$ without hypoglycaemia should not be automatically switched to a biosimilar insulin. Following the switch to a biosimilar insulin, it is recommended that provision for review and ongoing supervision by a specialist team is provided. With the advent of an increasingly complex portfolio of insulin therapy, it is imperative that all healthcare staff receive education about safe insulin prescribing which specifically includes information on biosimilar insulin.

\section{Key practical considerations for healthcare professionals}

- Change from one insulin to another should be done with supervision and consent of the prescribing healthcare professional in consultation with the patient.

- It is essential that insulin is prescribed by the brand name and that the device is specified. This information should be included in patient documentation (eg, insulin safety card/patient insulin passport)

- Patients can expect to see a similar blood glucose-lowering effect and safety profile as the reference product.

- The stability, shelf life and storage of the biosimilar insulin may differ from its reference product; therefore, the SPC should always be checked.

\section{Key messages}

- $A B C D$ acknowledges the benefit from the development of biosimilar insulins predicated upon potential cost savings to the NHS without compromising either efficacy or safety

- Patients established on a current insulin regimen achieving their target $\mathrm{HbA}_{1 \mathrm{c}}$ without hypoglycaemia should not be automatically switched to a biosimilar insulin

- $A B C D$ supports the position that biosimilar insulins could be considered for all newly diagnosed patients with type 1 diabetes who have not been exposed to the reference drug and in patients with type 1 diabetes who require a review of their therapy due to poor control

- A switch to a biosimilar insulin should only be made by clinical teams who have training, expertise and experience in treating people with diabetes

- With the advent of an increasingly complex portfolio of insulin therapy it is imperative that all healthcare staff receive education about safe insulin prescribing which specifically includes information on biosimilar insulin

- The insulin pen delivery device may be unique to the biosimilar insulin. Patients will need to be instructed on the correct use of the biosimilar pen device.

Conflict of interest None declared. Funding None.

\section{References}

1. Heinemann L, Hompesch M. Biosimilar insulins: basic considerations. J Diabetes Sci Technol 2014;8(1):6-13. https://doi.org/10.1177/1932296813516958

2. Johnson IS. Human insulin from recombinant DNA technology. Science 1983;219(4585):632-7. https://doi.org/10.1126/science.6337396

3. Llano A, Fisher M, McKay G. Biosimilar insulin: the current landscape. Pract Diabetes 2017;34(2):51-4. https://doi.org/10.1002/pdi.2079

4. Committee for Medicinal Products for Human Use. Guideline on similar biological medicinal products. http://www.ema.europa.eu/docs/en_GB/document_library/Scientific_guideline/ 2013/05/NC500142978.pdf (accessed 24 Feb 2018).

5. NICE. Position Statement: Evaluating biosimilar medicines. January 2015. https://www.nice.org.uk/news/article/evaluating-biosimilar-medicines (accessed 24 Feb 2018).

6. Blevins TC, Dahl D, Rosenstock J, et al. Efficacy and safety of LY2963016 insulin glargine compared with insulin glargine (Lantus $\left.{ }^{\circledR}\right)$ in patients with type 1 diabetes in a randomized controlled trial: the ELEMENT 1 study. Diabetes Obes Metab 2015;17(8):726-33.

https://doi.org/10.1111/dom.12496

7. Rosenstock J, Hollander P, Bhargava A, et al. Similar efficacy and safety of LY2963016 insulin glargine and insulin glargine (Lantus ${ }^{\circledR}$ ) in patients with type 2 diabetes who were insulin-naive or previously treated with insulin glargine: a randomized, double-blind controlled trial (the ELEMENT 2 study). Diabetes Obes Metab 2015;17(8):734-41.

https://doi.org/10.1111/dom.12482 
8. European Medicines Agency. Summary of the European public assessment report (EPAR) for Lusduna. http://www.ema.europa.eu/docs/en_GB/document_library/EPAR_-_Public_assessment_report/human/ 004101 NC500219588. pdf (accessed 24 Feb 2018).

9. European Medicines Agency. Summary of the European public assessment report (EPAR) for Insulin Lispro Sanofi. http://www.ema.europa.eu/ docs/en_GB/document_library/EPAR_-_Public_assessment_report/ human/004303NWC500235297.pdf (accessed 24 Feb 2018).

10. Committee for Medicinal Products for Human Use (CHMP) Summary of opinion, Semglee. http://www.ema.europa.eu/docs/en_GB/document_li-
brary/Summary_of_opinion_-_Initial_authorisation/human/004280/ WC500242403.pdf (accessed 24 Feb 2018).

11. NICE. Evidence summary: Diabetes mellitus type 1 and type 2: insulin glargine biosimilar (Abasaglar). Dec 2015. https://www.nice.org.uk/guidance/esnm64 (accessed 24 Feb 2018)

12. European Medicines Agency. Summary of the European public assessment report (EPAR) for Abasaglar. https://www.ema.europa.eu/documents/ overview/abasaglar-previously-abasria-epar-summary-public_en.pdf (accessed 24 Feb 2018). 